\title{
Mixed Matrix Membranes (MMMs) Comprising Exfoliated 2D Covalent Organic Frameworks (COFs) for Efficient $\mathrm{CO}_{2}$ Separation
}

Zixi Kang ${ }^{\dagger, \|}$, Yongwu Peng ${ }^{\dagger, \|}$, Yuhong Qian ${ }^{\dagger, \|}$, Daqiang Yuan ${ }^{\ddagger}$, Matthew A. Addicoat ${ }^{\S}$, Thomas Heine ${ }^{\S}$, Zhigang $\mathrm{Hu}^{\dagger}$, Lincoln Tee ${ }^{\dagger}$, Zhengang $\mathrm{Guo}^{\dagger}$, and Dan Zhao ${ }^{\dagger, *}$

${ }^{\dagger}$ Department of Chemical and Biomolecular Engineering, National University of Singapore, 4 Engineering Drive 4, 117585, Singapore

${ }^{\ddagger}$ State Key Laboratory of Structural Chemistry, Fujian Institute of Research on the Structure of Matter, Chinese Academy of Sciences, Fuzhou, 350002 Fujian, China

${ }^{\S}$ School of Engineering and Science, Jacobs University Bremen, Campus Ring 1, 28759 Bremen, Germany

|| These authors contributed equally to this work.

Correspondence and requests for materials should be addressed to D.Z. (E-mail: chezhao@nus.edu.sg). 


\section{Experimental Details}

\section{Calculations of isosteric heat of adsorption $\left(Q_{s t}\right)$}

The $\mathrm{CO}_{2}$ adsorption isotherms measured at $273 \mathrm{~K}$ and $298 \mathrm{~K}$ were first fitted to a virial equation (Equation 1). The fitting parameters were then used to calculate the isosteric heat of adsorption $\left(Q_{s t}\right)$ using Equation 2,

$$
\begin{aligned}
& \ln P=\ln N+\frac{1}{T} \sum_{i=0}^{m} a_{i} N^{i}+\sum_{i=0}^{n} b_{i} N^{i} \\
& Q_{s t}=-R \sum_{i=0}^{m} a_{i} N^{i}
\end{aligned}
$$

where $P$ is pressure (mmHg), $N$ is adsorbed quantity $\left(\mathrm{mmol} \mathrm{g}^{-1}\right), T$ is temperature $(\mathrm{K}), R$ is gas constant (8.314 $\left.\mathrm{J} \mathrm{K}^{-1} \mathrm{~mol}^{-1}\right), a_{i}$ and $b_{i}$ are virial coefficients, $m$ and $n$ represent the number of coefficients required to adequately describe the isotherms (herein, $m=5, n=2$ ).

\section{Ideal Adsorption Solution Theory (IAST) Selectivity}

The $\mathrm{CO}_{2}, \mathrm{CH}_{4}$, and $\mathrm{H}_{2}$ adsorption isotherms were first fitted to a dual-site Langmuir-Freundlich (DSLF) model (Equation 3),

$$
q=\frac{q_{\text {sat }, A} b_{A} p^{\alpha_{A}}}{1+b_{A} p^{\alpha_{A}}}+\frac{q_{s a t, B} b_{B} p^{\alpha_{B}}}{1+b_{B} p^{\alpha_{B}}}
$$

where $q$ is the amount of adsorbed gas ( $\left.\mathrm{mmol} \mathrm{g}^{-1}\right), p$ is the bulk gas phase pressure (bar), $q_{\text {sat }}$ is the saturation amount (mmol $\left.\mathrm{g}^{-1}\right), b$ is the Langmuir-Freundlich parameter $\left(\operatorname{bar}^{-\alpha}\right), \alpha$ is the Langmuir-Freundlich exponent (dimensionless) for two adsorption sites A and B indicating the presence of weak and strong adsorption sites.

IAST starts from the Raoults' Law type of relationship between fluid and adsorbed phase,

$P_{i}=P y_{i}=P_{i}^{o} x_{i}$

$\sum_{i=1}^{n} x_{i}=\sum_{i=1}^{n} \frac{P_{i}}{P_{i}^{0}}=1$

where $P_{i}$ is partial pressure of component $i$ (bar), $P$ is total pressure (bar), $y_{i}$ and $x_{i}$ represent mole fractions of component $i$ in gas and adsorbed phase (dimensionless). $P_{i}^{0}$ is equilibrium vapour pressure (bar).

In IAST, $P_{i}^{0}$ is defined by relating to spreading pressure $\pi$, 
$\frac{\pi S}{R T}=\int_{0}^{P_{i}^{0}} \frac{q_{i}\left(P_{i}\right)}{P_{i}} d P_{i}=\Pi$ (Constant)

where $\pi$ is spreading pressure, $S$ is specific surface area of adsorbent $\left(\mathrm{m}^{2} \mathrm{~g}^{-1}\right), R$ is gas constant (8.314 $\mathrm{J} \mathrm{K}^{-1} \mathrm{~mol}^{-1}$ ), $T$ is temperature $(\mathrm{K}), q_{i}\left(P_{i}\right)$ is the single component equilibrium obtained from isotherm $\left(\mathrm{mmol} \mathrm{g}^{-1}\right)$.

For a dual-site Langmuir-Freundlich (DSLF) model, we have an analytical expression for the integral:

$\int_{0}^{P_{i}^{0}} \frac{q_{i}\left(P_{i}\right)}{P_{i}} d P_{i}=\Pi$ (Constant) $=\frac{q_{\text {sat }, A}}{\alpha_{A}} \ln \left[1+b_{A}\left(P_{i}^{0}\right)^{\alpha_{A}}\right]+\frac{q_{s a t, B}}{\alpha_{B}} \ln \left[1+b_{B}\left(P_{i}^{0}\right)^{\alpha_{B}}\right]$

The isotherm parameters are derived from the previous fitting. For a binary component system the unknowns will be $\Pi, P_{1}{ }^{0}$, and $P_{2}{ }^{0}$ which can be obtained by simultaneously solving Equations 5 and 7.

The adsorbed amount for each compound in a mixture is

$q_{i}^{m i x}=x_{i} q_{T}$

$\frac{1}{q_{T}}=\sum_{i=1}^{n} \frac{x_{i}}{q_{i}\left(P_{i}^{o}\right)}$

where $q_{i}^{\text {mix }}$ is the adsorbed amount of component $i\left(\mathrm{mmol} \mathrm{g}^{-1}\right), q_{T}$ is the total adsorbed amount $\left(\mathrm{mmol} \mathrm{g}^{-1}\right)$.

The adsorption selectivities $S_{a d s}$ were calculated using Equation 10 .

$S_{\text {ads }}=\frac{q_{1} / q_{2}}{p_{1} / p_{2}}$

In this study, IAST calculations were carried out assuming a $\mathrm{CO}_{2} / \mathrm{CH}_{4}$ or $\mathrm{CO}_{2} / \mathrm{H}_{2}$ binary mixed gas with a molar ratio of 50:50 at $273 \mathrm{~K}$ and pressures up to 1 bar. 

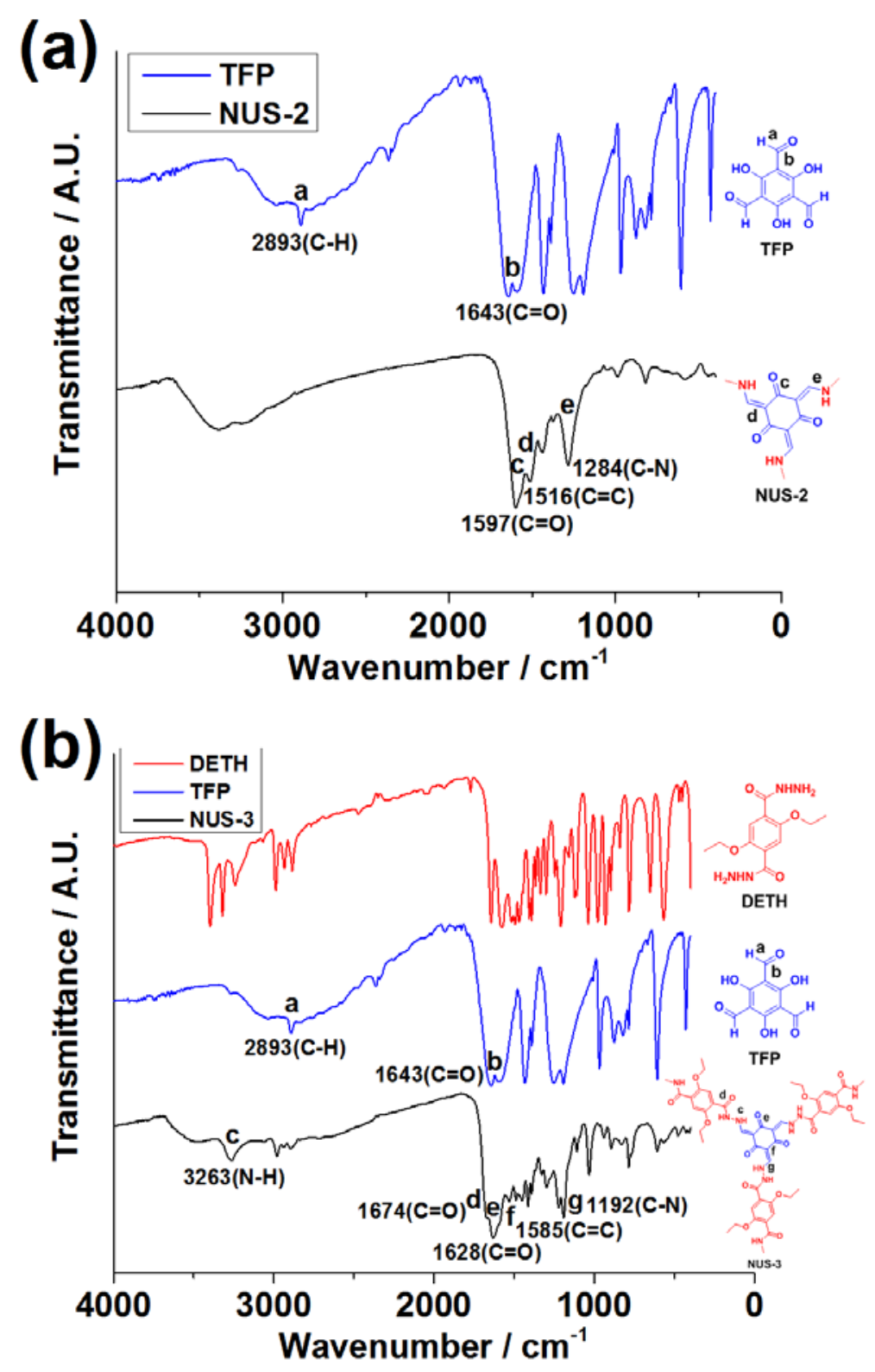

Figure S1. FTIR spectra of (a) NUS-2 and (b) NUS-3. 


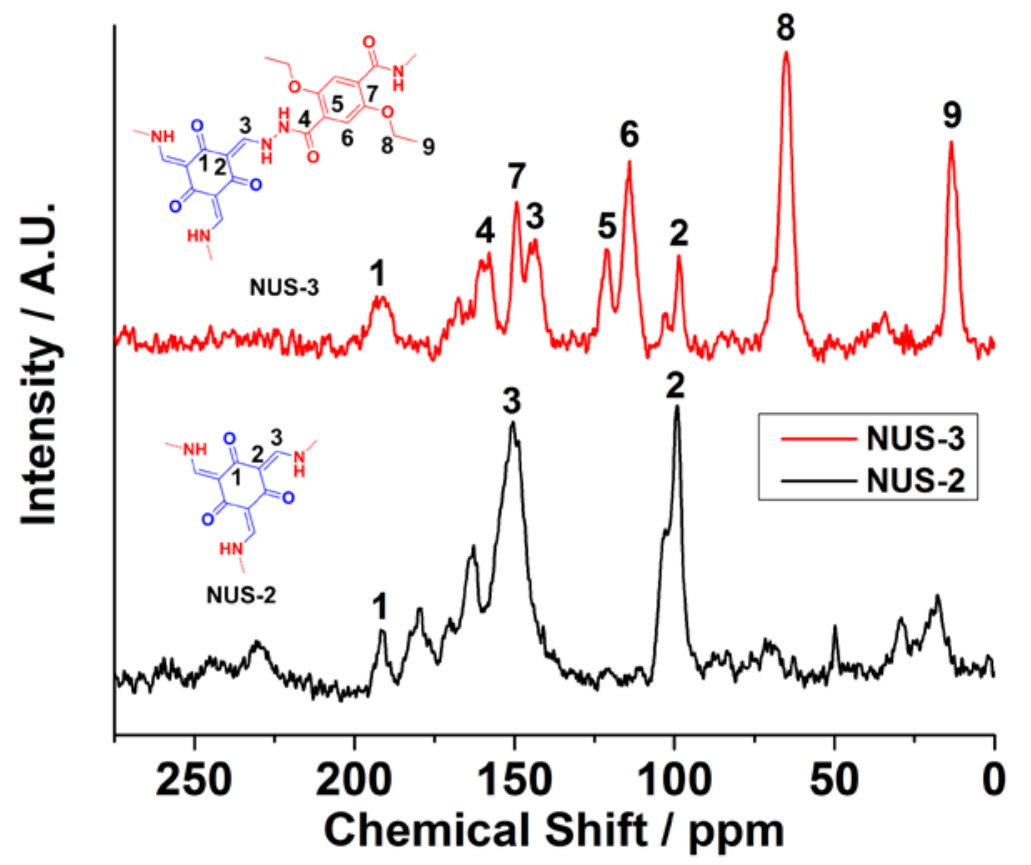

Figure S2. ${ }^{13} \mathrm{C}$ CP/MAS solid-state NMR spectra of NUS-2 (black) and NUS-3 (red). 

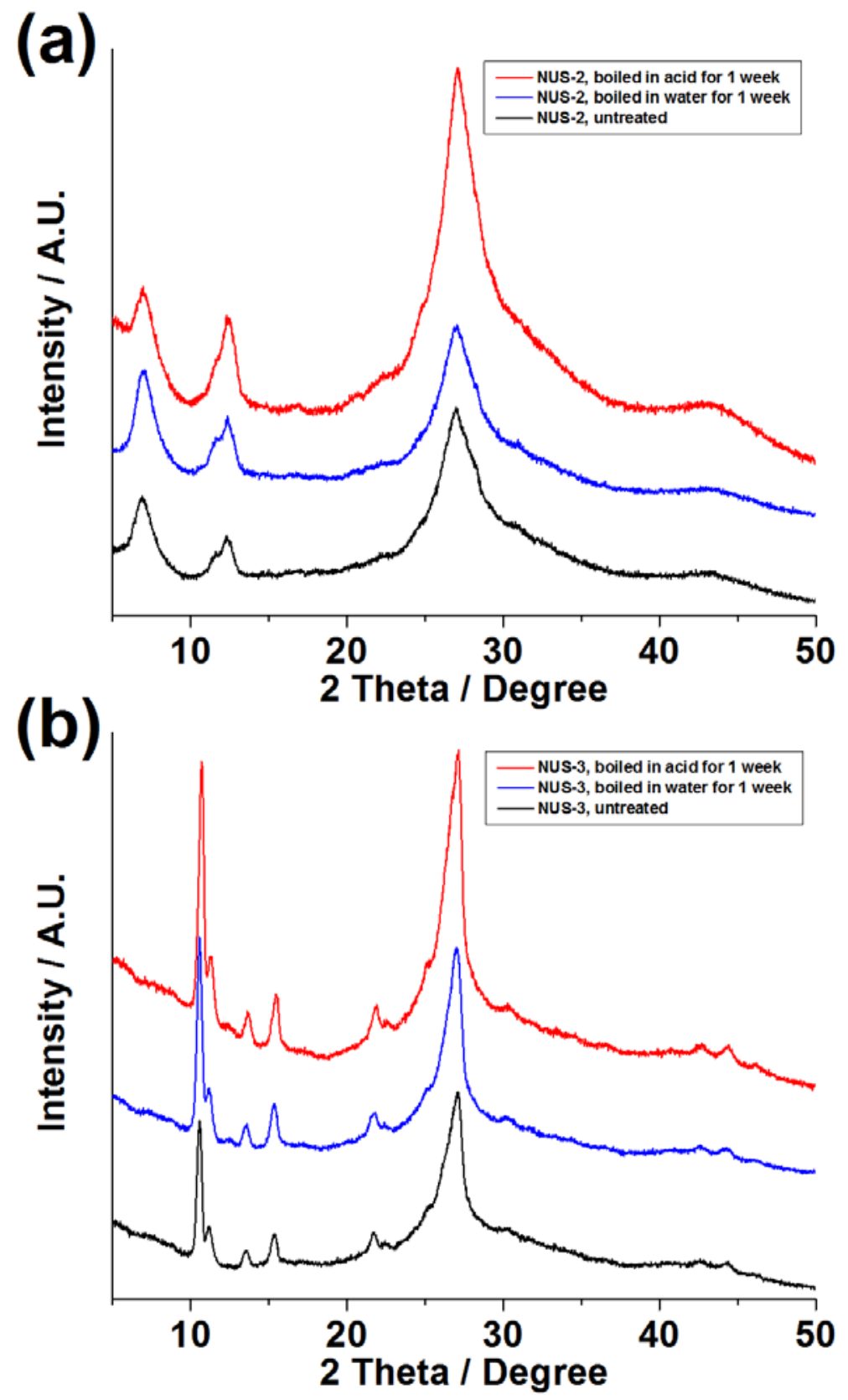

Figure S3. PXRD patterns of (a) NUS-2 and (b) NUS-3 under various treating conditions. 


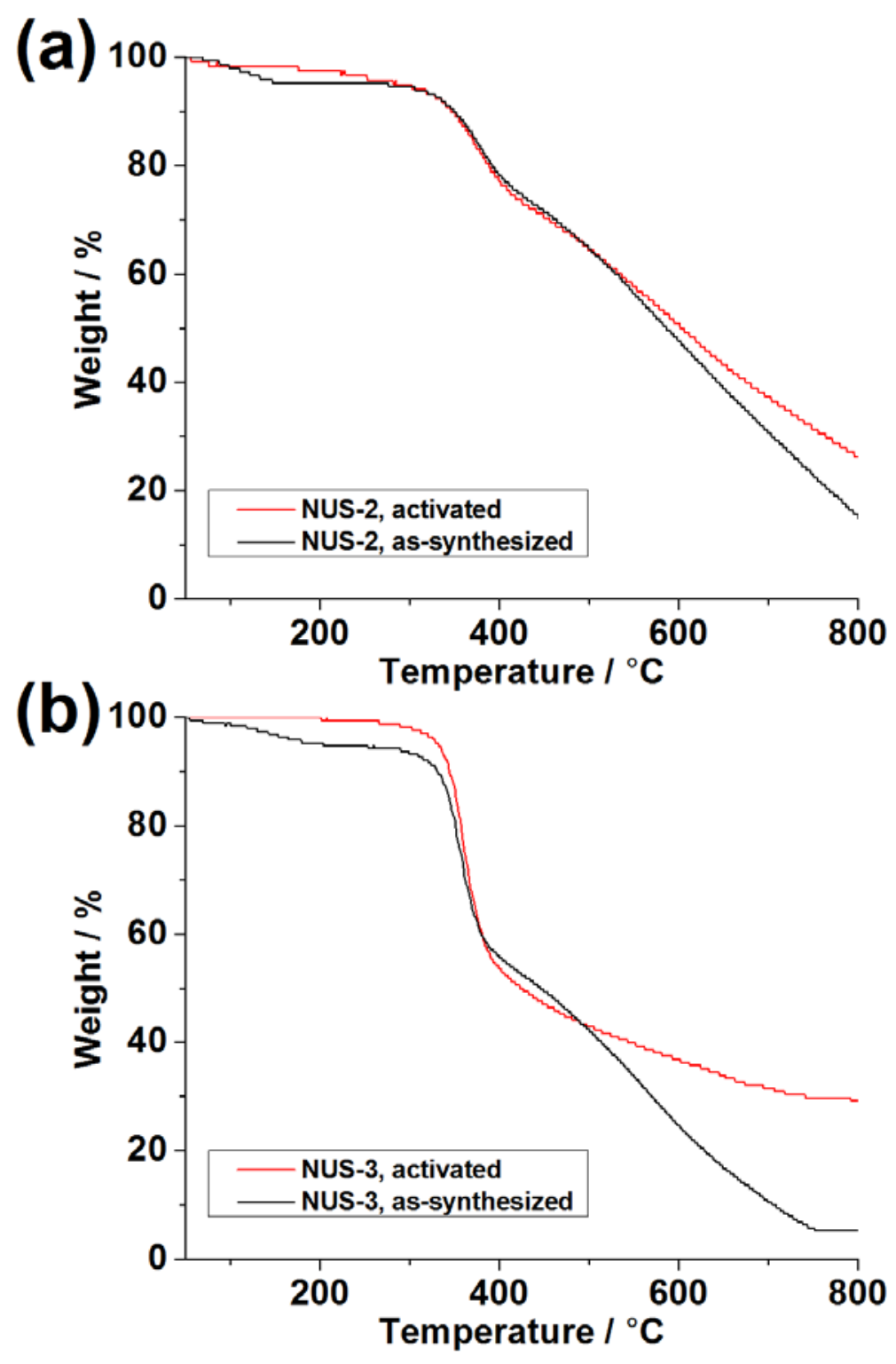

Figure S4. TGA curves of (a) NUS-2 and (b) NUS-3. 


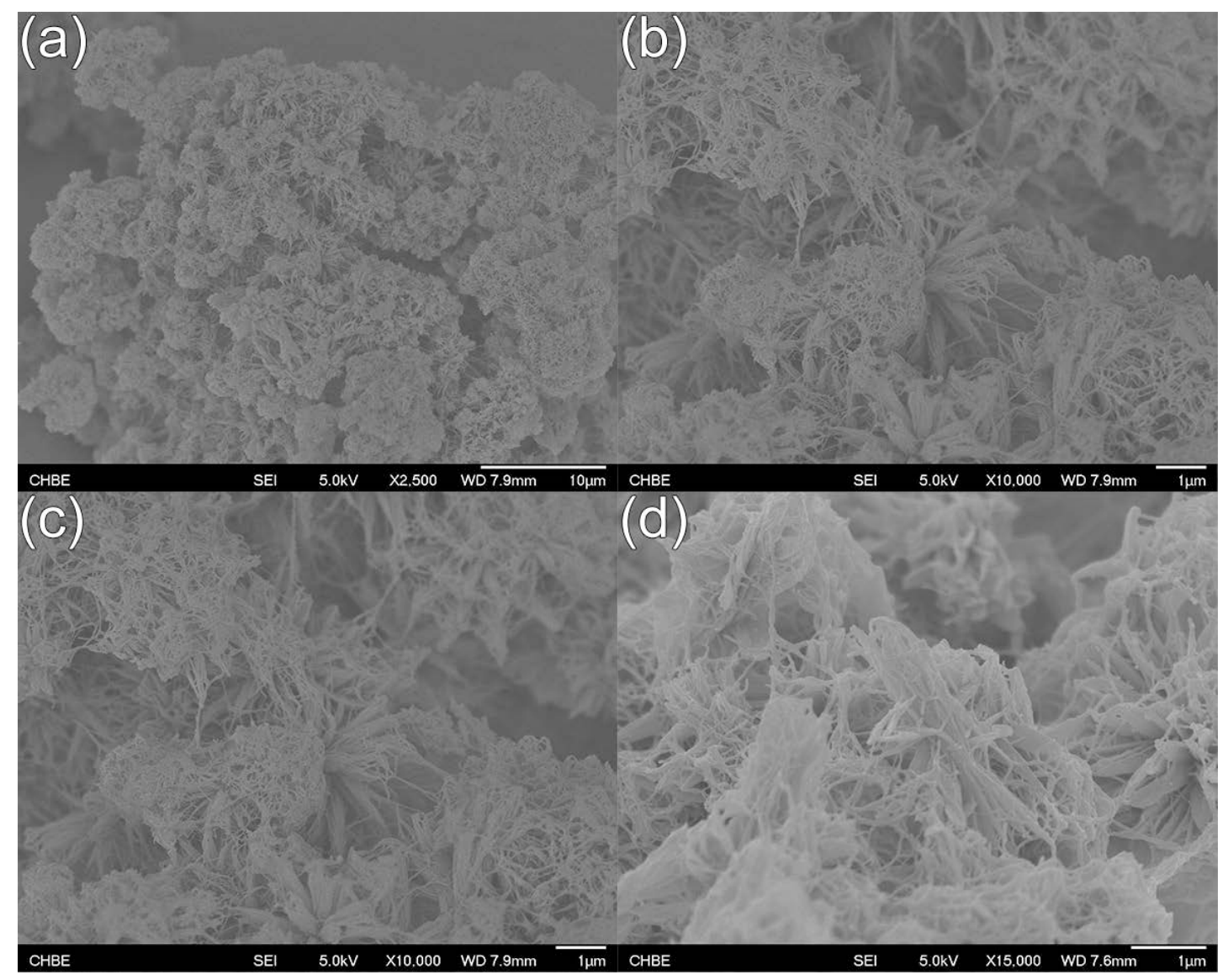

Figure S5. FE-SEM images of (a, b) NUS-2 and (c, d) NUS-3. 


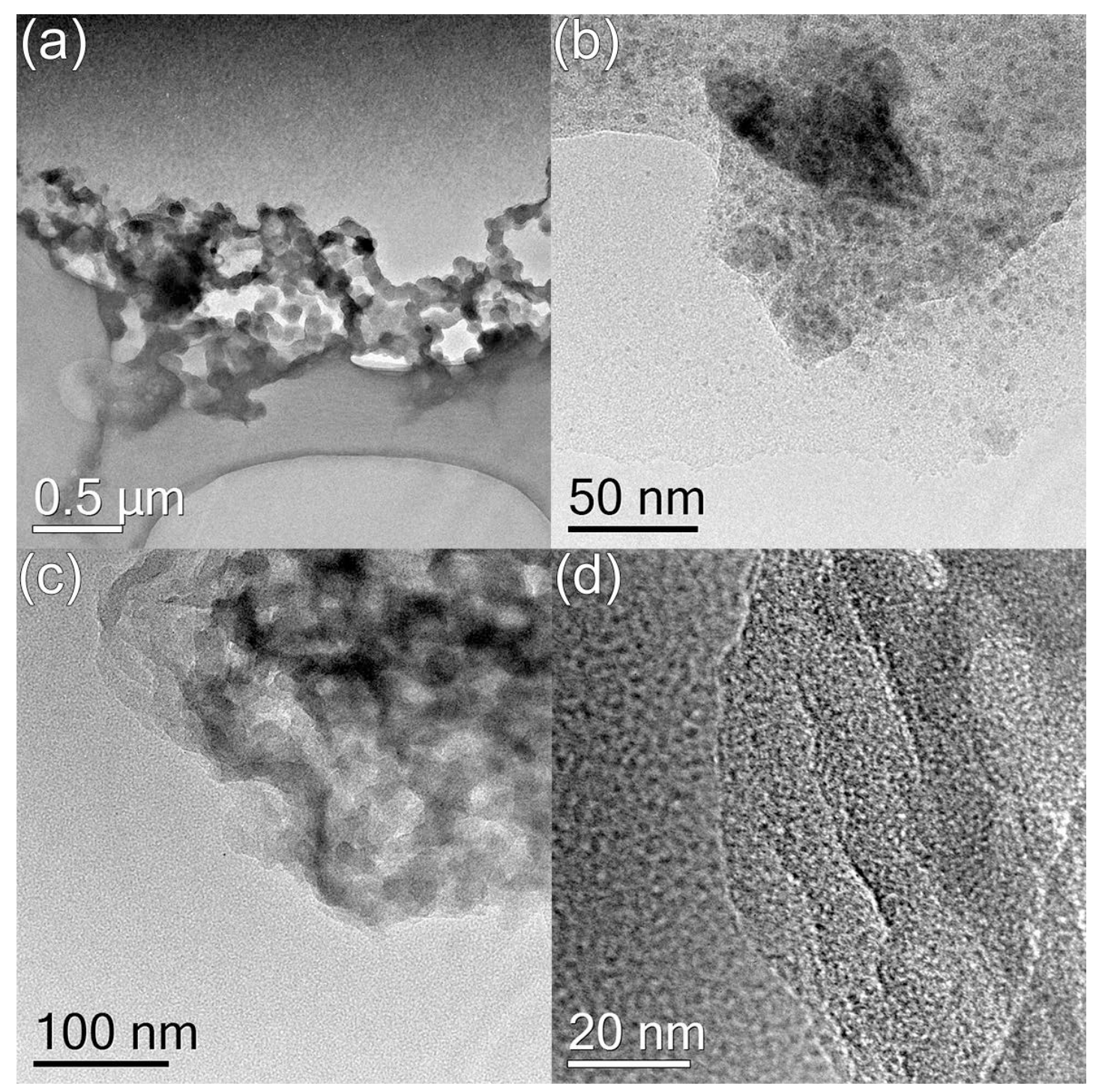

Figure S6. FE-TEM images of (a, b) NUS-2 and (c, d) NUS-3. 
(a)

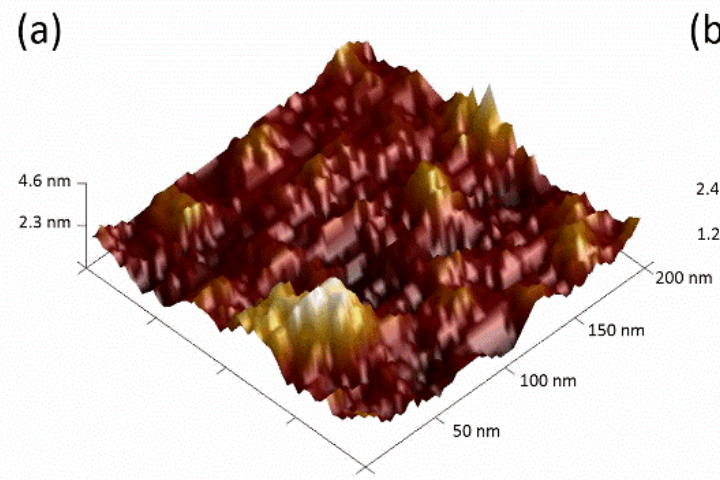

(b)

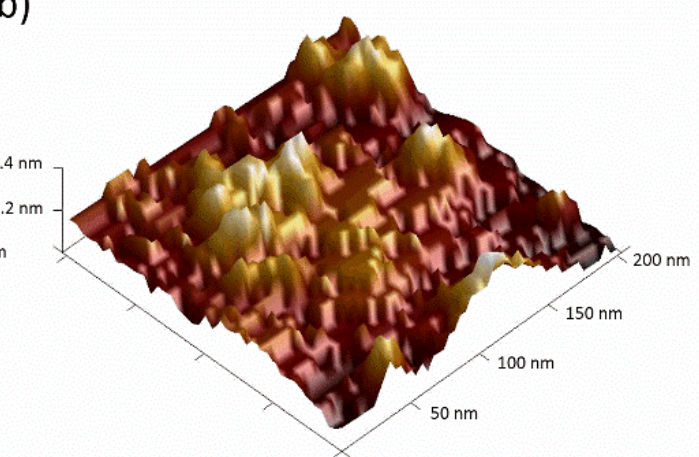

Figure S7. Typical AFM phase images of (a) NUS-2 and (b) NUS-3. 

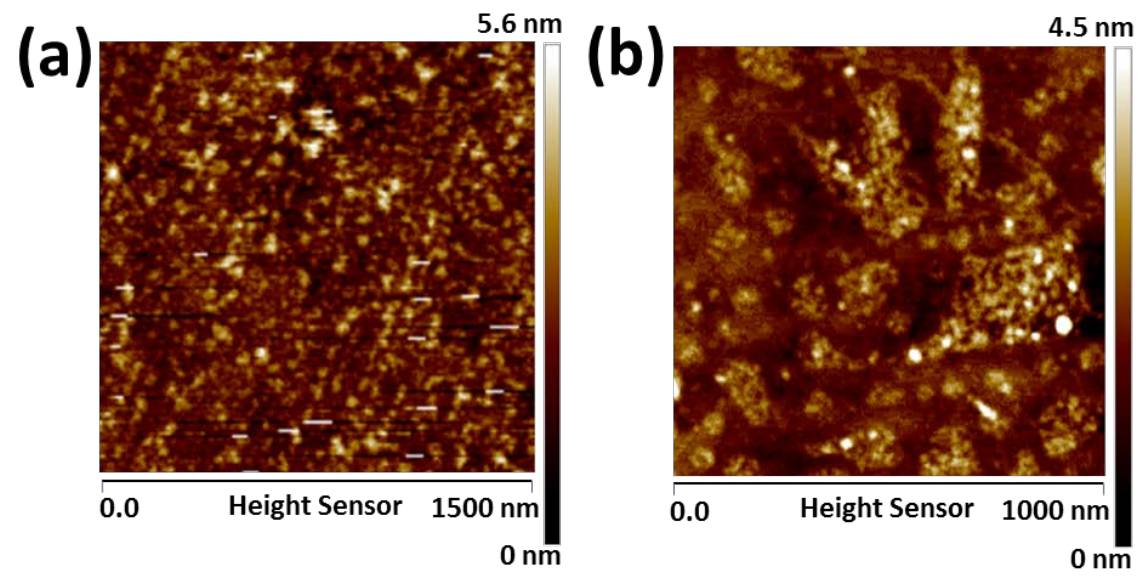

Figure S8. Large scale AFM images of (a) NUS-2 and (b) NUS-3. The statistical distribution of nanosheets' dimension and height is listed in Table S5 and S6. 
(a)

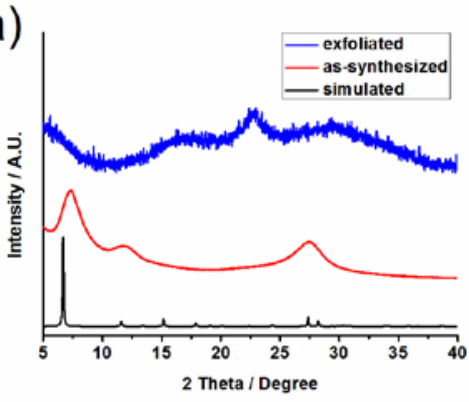

(c)

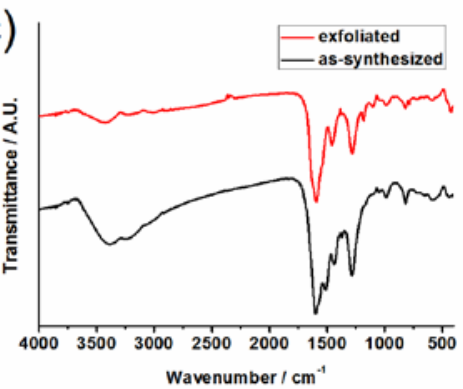

(b)

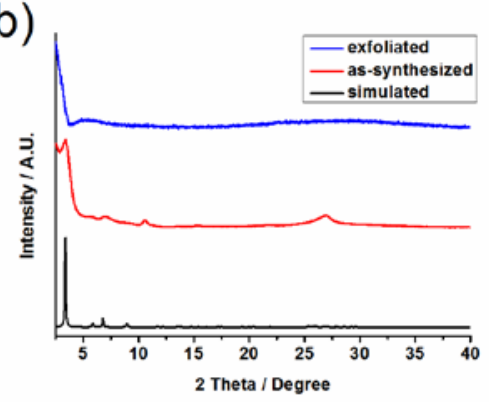

(d)

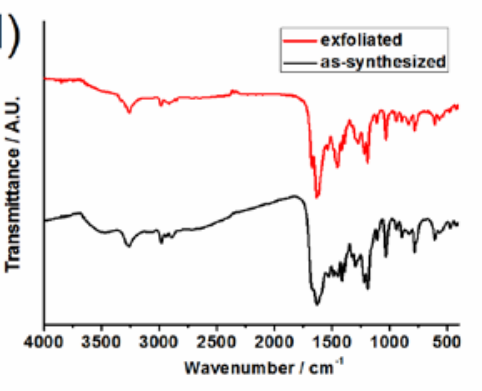

Figure S9. (a, b) PXRD and (c, d) FTIR spectra of exfoliated NUS-2 and exfoliated NUS-3, respectively. 


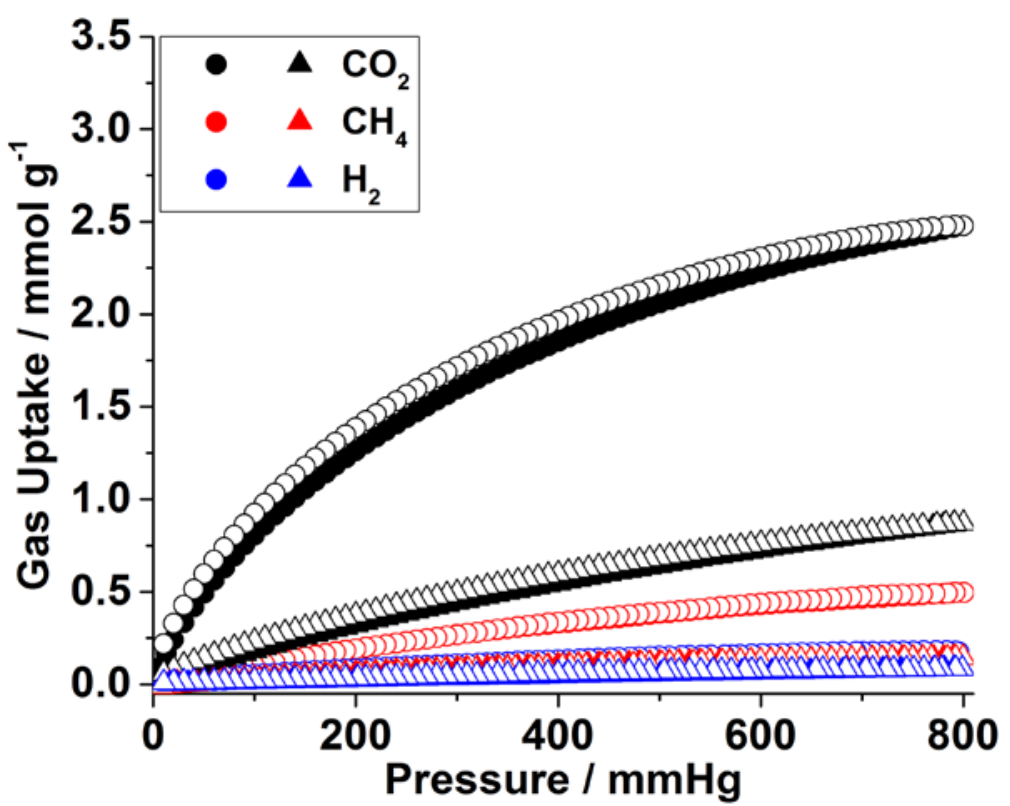

Figure S10. $\mathrm{CO}_{2}$ (black), $\mathrm{CH}_{4}$ (red), and $\mathrm{H}_{2}$ (blue) sorption isotherms of NUS-2 (circle) and NUS-3 (triangle) at $298 \mathrm{~K}$. Adsorption, closed; desorption, open. 


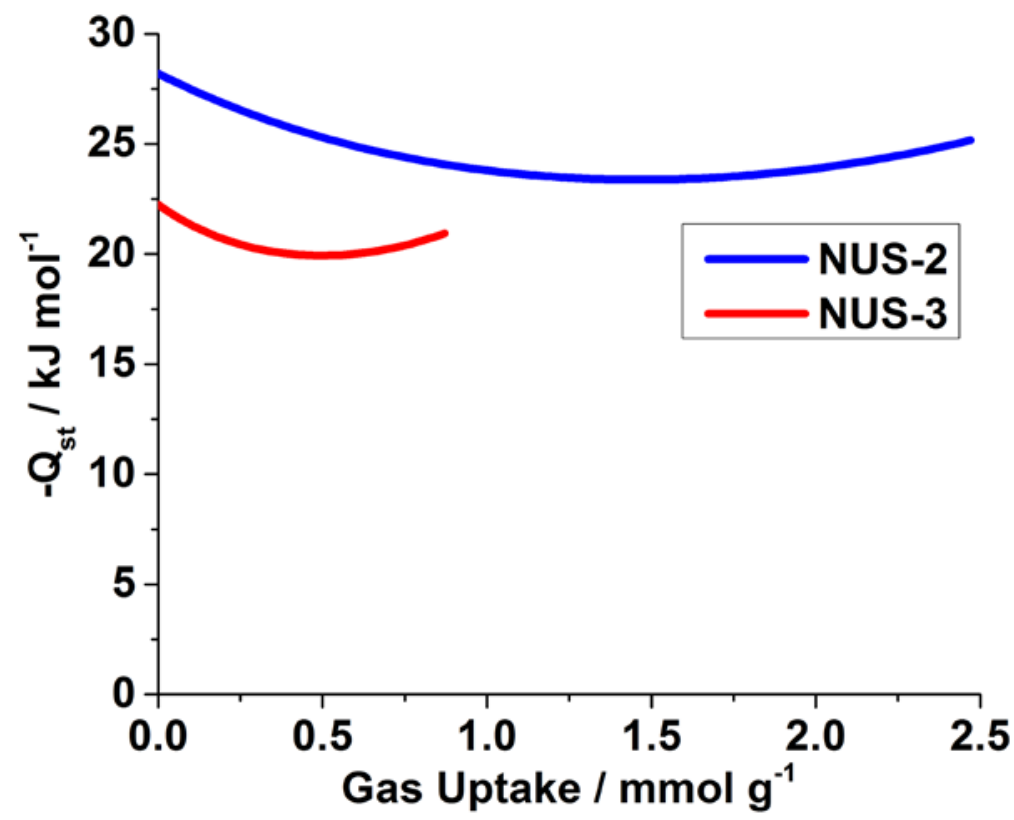

Figure S11. Isosteric heat of adsorption $\left(\mathrm{Q}_{\mathrm{st}}\right)$ of $\mathrm{CO}_{2}$ in NUS-2 (blue) and NUS-3 (red). 
(a)
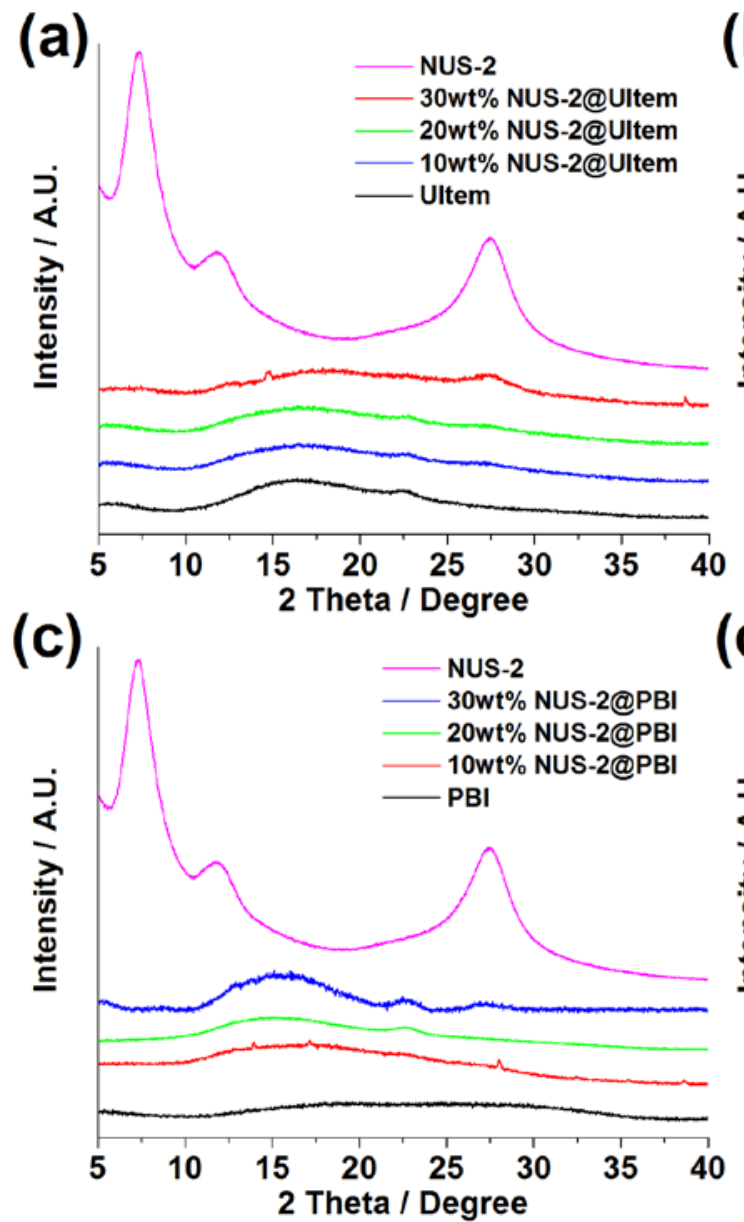

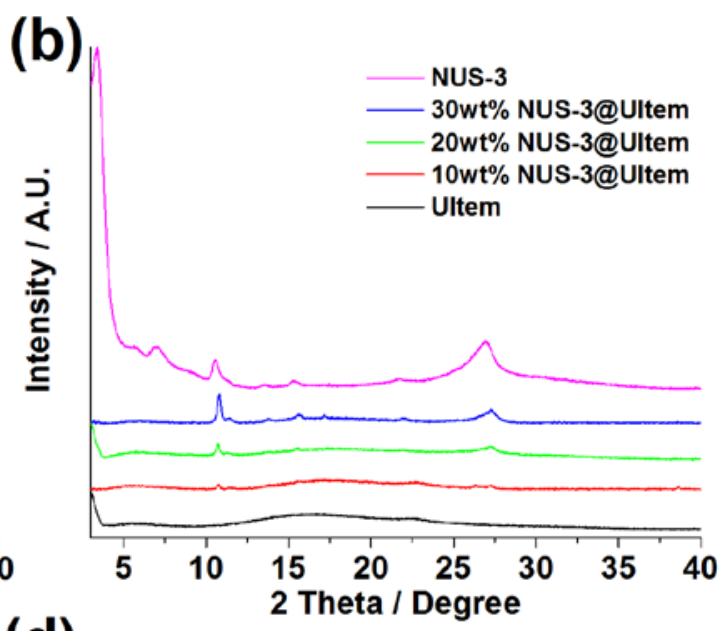

(d)

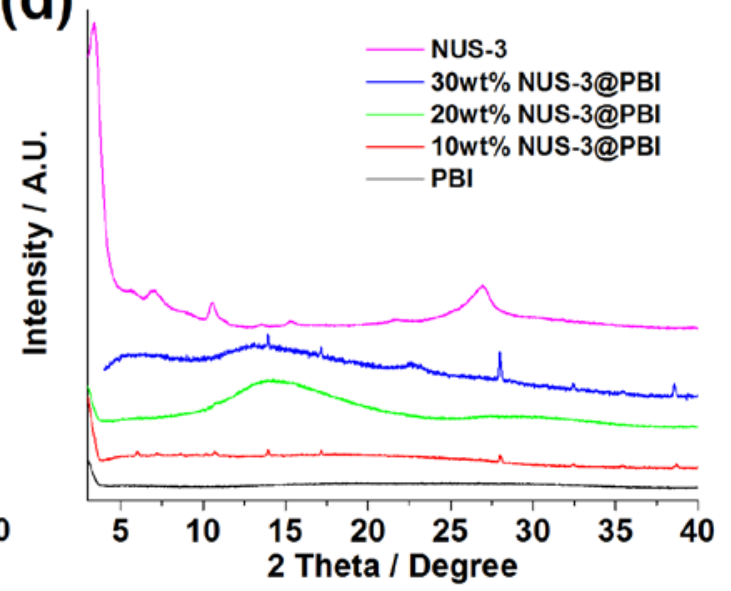

Figure S12. PXRD patterns of (a, b) Ultem and (c, d) PBI along with MMMs and COFs. 


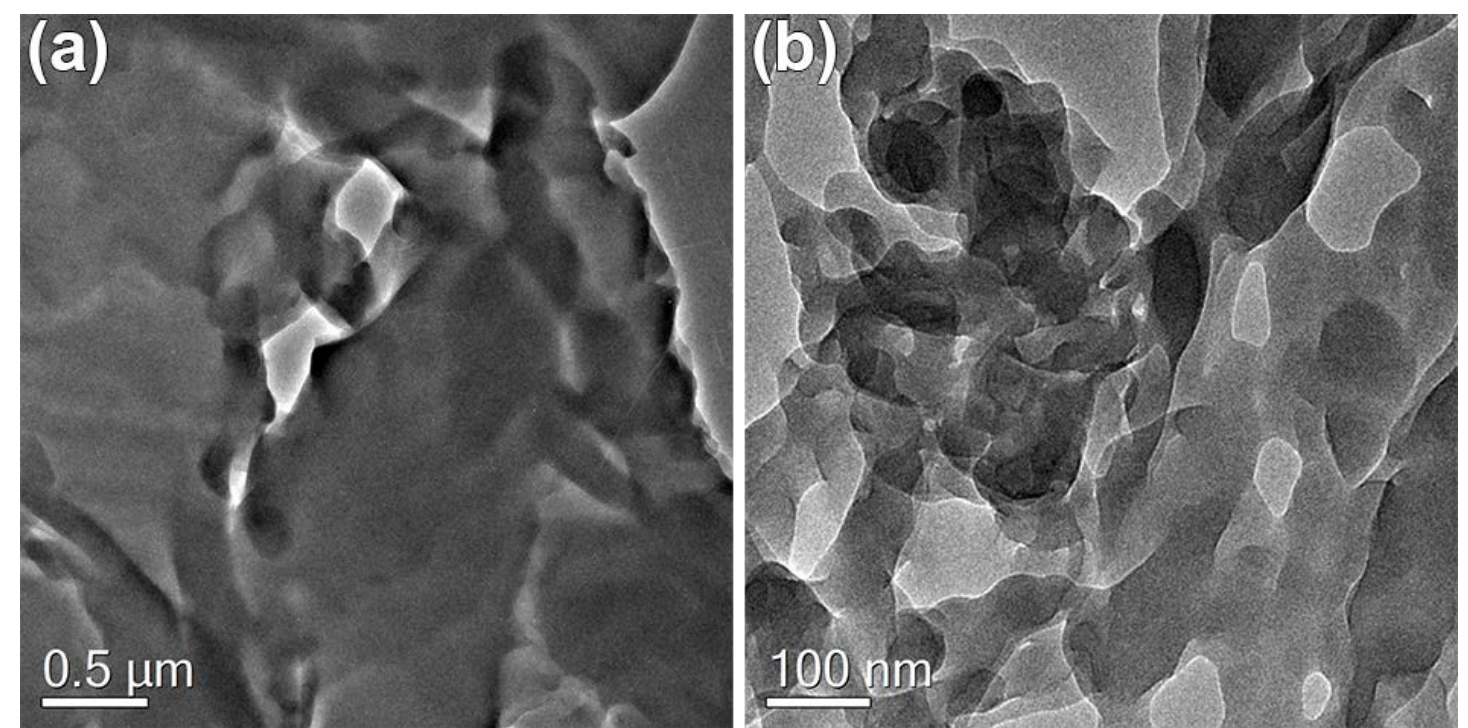

Figure S13. FE-TEM images of (a) pure PBI membrane and (b) 20wt\%NUS-3@PBI MMM. 


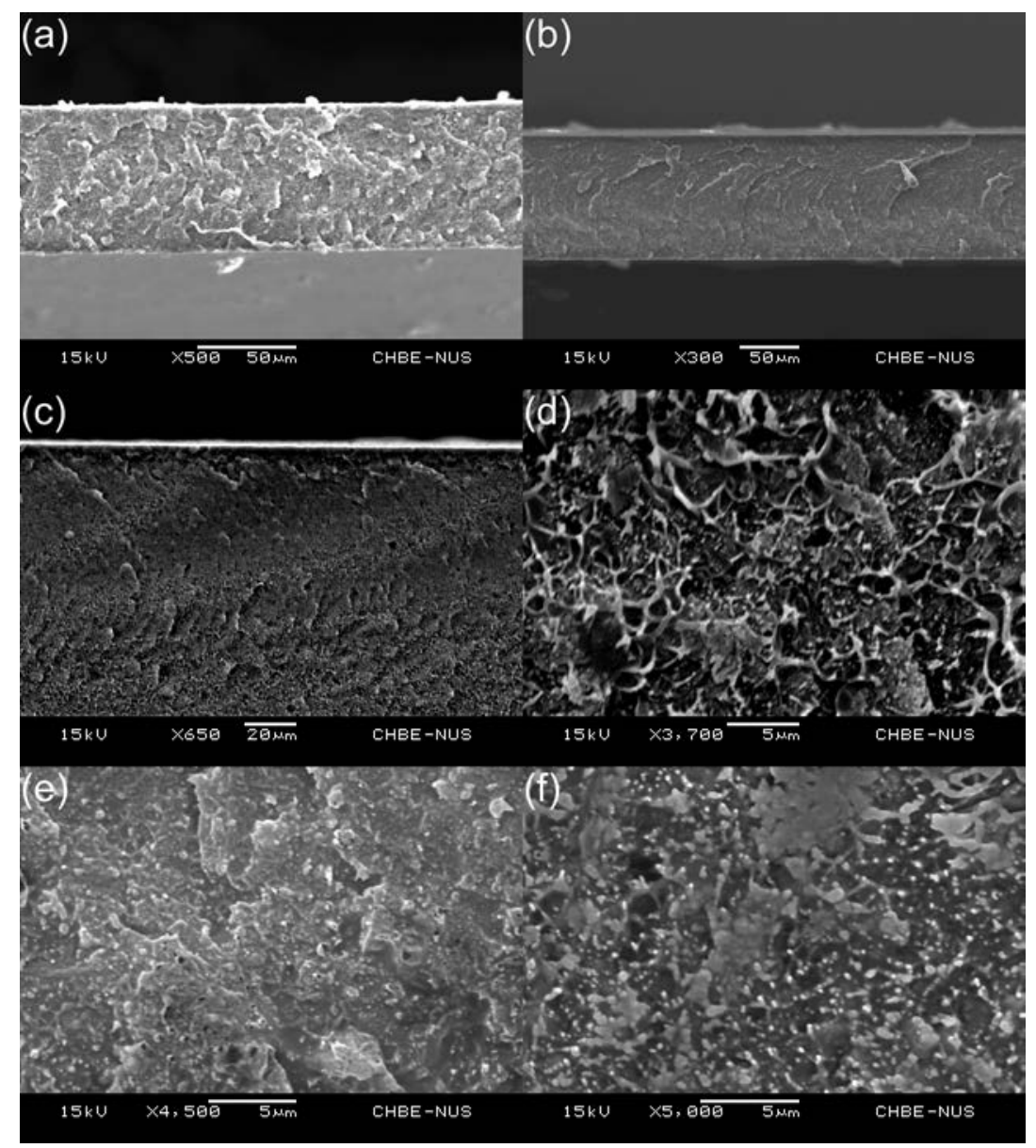

Figure S14. (a, c, e) Cross-sectional SEM images of 10-30wt\% NUS-2@Ultem; (b, d, f) cross-sectional SEM images of 10-30wt\% NUS-3@Ultem. 


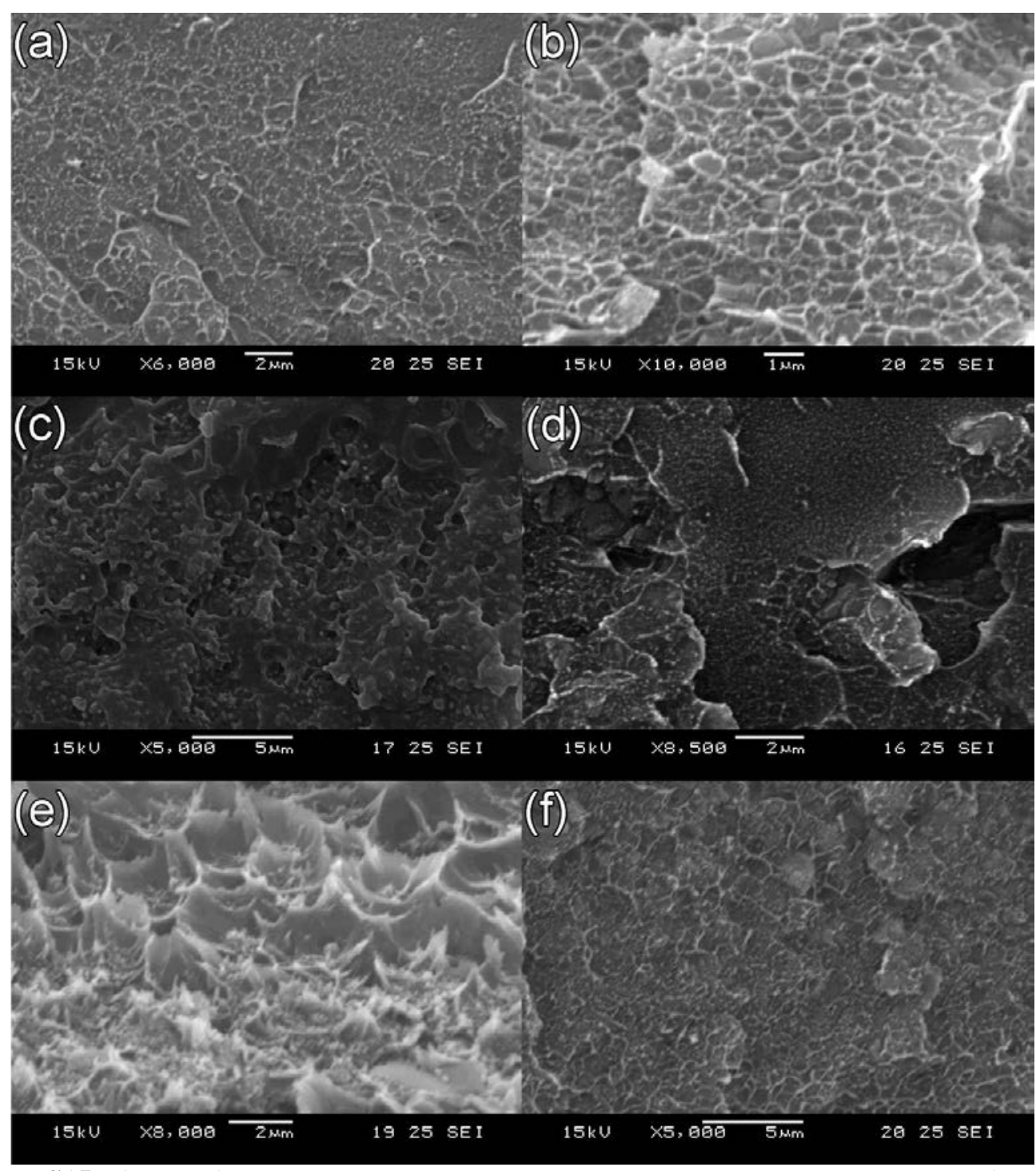

Figure S15. (a, c, e) Cross-sectional SEM images of 10-30wt\% NUS-2@PBI; (b, d, f) cross-sectional SEM images of 10-30wt\% NUS-3@PBI. 
Table S1. Crystallographic information of modelled NUS-2.

\begin{tabular}{|l|l|}
\hline Parameter & $\mathrm{NUS}-2$ \\
\hline Empirical formula & $\mathrm{C}_{9} \mathrm{H}_{6} \mathrm{~N}_{3} \mathrm{O}_{3}$ \\
\hline $\mathrm{F}_{\mathrm{w}}$ & 204.16 \\
\hline Stacking method & slipped-AA \\
\hline Crystal system & Hexagonal \\
\hline Space group & $P 6 / \mathrm{m}$ \\
\hline$a[\AA]$ & 15.3195 \\
\hline$b[\AA]$ & 15.2892 \\
\hline$c[\AA]$ & 6.50778 \\
\hline$\alpha[$ deg] & 90 \\
\hline$\beta$ [deg] & 90 \\
\hline$\gamma$ [deg] & 59.7289 \\
\hline Total energy (a.u.) & -138.335771 \\
\hline Interlayer distance ( $\AA$ ) & 3.30 \\
\hline LJ dispersion (a.u.) & 0.3517 \\
\hline Stacking energy (kcal/mol) & 32.27 \\
\hline
\end{tabular}


Table S2. Atom sites for modelled NUS-2.

\begin{tabular}{|c|c|c|c|}
\hline Atom site label & Atom site fract $\mathrm{x}$ & Atom site fract $\mathrm{y}$ & Atom site fract $\mathrm{z}$ \\
\hline C1 & 0.12578 & 0.70899 & 0.01847 \\
\hline C2 & 0.02699 & 0.66115 & 0.01847 \\
\hline C3 & 0.21428 & 0.52147 & 0.01847 \\
\hline O1 & 0.19228 & 0.77428 & 0.01847 \\
\hline O2 & 0.93468 & 0.68795 & 0.01847 \\
\hline O3 & 0.27991 & 0.42912 & 0.01847 \\
\hline C4 & 0.24197 & 0.60068 & 0.01847 \\
\hline C5 & 0.05803 & 0.73768 & 0.01847 \\
\hline C6 & 0.10595 & 0.5532 & 0.01847 \\
\hline C7 & 0.34325 & 0.57584 & 0.01847 \\
\hline H1 & 0.36274 & 0.63632 & 0.01847 \\
\hline C8 & 0.08074 & 0.47692 & 0.01847 \\
\hline H2 & 0.14053 & 0.39624 & 0.01847 \\
\hline C9 & 0.98156 & 0.83964 & 0.01847 \\
\hline H3 & 0.90104 & 0.86119 & 0.01847 \\
\hline N1 & 0.41878 & 0.47919 & 0.01847 \\
\hline H4 & 0.39859 & 0.42238 & 0.01847 \\
\hline N2 & 0.98393 & 0.49928 & 0.01847 \\
\hline N3 & 0.00397 & 0.91391 & 0.01847 \\
\hline H5 & 0.92733 & 0.57695 & 0.01847 \\
\hline H6 & 0.08177 & 0.89152 & 0.01847 \\
\hline & & & \\
\hline
\end{tabular}


Table S3. Crystallographic information of modelled NUS-3.

\begin{tabular}{|l|l|}
\hline Parameter & $\mathrm{NUS}-3$ \\
\hline Empirical formula & $\mathrm{C}_{9} \mathrm{H}_{9} \mathrm{~N}_{2} \mathrm{O}_{3}$ \\
\hline $\mathrm{F}_{\mathrm{w}}$ & 193.18 \\
\hline Stacking method & slipped-AA \\
\hline Crystal system & Hexagonal \\
\hline Space group & $P 6 / \mathrm{m}$ \\
\hline$a[\AA]$ & 30.1441 \\
\hline$b[\AA]$ & 30.1305 \\
\hline$c[\AA]$ & 7.08186 \\
\hline$\alpha[$ deg] & 90.2291 \\
\hline$\beta$ [deg] & 90.3233 \\
\hline$\gamma$ [deg] & 60.4944 \\
\hline Total energy (a.u.) & -401.6527 \\
\hline Interlayer distance ( $\AA$ ) & 3.88 \\
\hline LJ dispersion (a.u.) & 1.0342 \\
\hline Stacking energy (kcal/mol) & 81.90 \\
\hline
\end{tabular}


Table S4. Atom sites for modelled NUS-3.

\begin{tabular}{|c|c|c|c|}
\hline Atom site label & Atom site fract $\mathrm{x}$ & Atom site fract $\mathrm{y}$ & Atom site fract $\mathrm{z}$ \\
\hline C1 & 0.16763 & 0.71211 & 0.09845 \\
\hline C2 & 0.10158 & 0.68271 & 0.0426 \\
\hline C3 & 0.19391 & 0.61719 & 0.13301 \\
\hline O1 & 0.17897 & 0.74693 & 0.10641 \\
\hline O2 & 0.05652 & 0.6937 & 0.00617 \\
\hline O3 & 0.22708 & 0.57196 & 0.16478 \\
\hline C4 & 0.20601 & 0.65907 & 0.14428 \\
\hline C5 & 0.116 & 0.72285 & 0.04459 \\
\hline C6 & 0.14153 & 0.62983 & 0.08046 \\
\hline C7 & 0.25528 & 0.64941 & 0.18576 \\
\hline H1 & 0.26405 & 0.68097 & 0.18818 \\
\hline C8 & 0.13165 & 0.58943 & 0.05609 \\
\hline H2 & 0.1619 & 0.54974 & 0.08193 \\
\hline C9 & 0.07894 & 0.77156 & 0.9876 \\
\hline H3 & 0.0405 & 0.77895 & 0.94593 \\
\hline N1 & 0.29315 & 0.6024 & 0.23086 \\
\hline H4 & 0.28592 & 0.57157 & 0.21505 \\
\hline N2 & 0.08557 & 0.59744 & $7.60 \mathrm{E}-04$ \\
\hline N3 & 0.91981 & 0.14428 & 0.83377 \\
\hline H5 & 0.05666 & 0.63514 & 0.97041 \\
\hline H6 & 0.94782 & 0.1366 & 0.93725 \\
\hline & & & \\
\hline
\end{tabular}


Table S5. Statistical distributions of nanosheet dimensions and heights of NUS-2

\begin{tabular}{|c|c|c|c|c|}
\hline \multirow{2}{*}{ Parameter } & \multicolumn{4}{|c|}{ NUS-2 } \\
\cline { 2 - 5 } & Mean & Min & Max & Sigma \\
\hline Total Count & 65 & 65 & 65 & 0 \\
\hline Height / nm & 3.4 & 1.3 & 15.5 & 3.3 \\
\hline Area / $\mathrm{nm}^{2}$ & 5509.5 & 1917.13 & 27913.4 & 5105.2 \\
\hline Diameter / nm & 77.9 & 49.4 & 188.5 & 30.8 \\
\hline
\end{tabular}

Table S6. Statistical distributions of nanosheet dimensions and heights of NUS-3

\begin{tabular}{|c|c|c|c|c|}
\hline \multirow{2}{*}{ Parameter } & \multicolumn{4}{|c|}{ NUS-3 } \\
\cline { 2 - 5 } & Mean & Min & Max & Sigma \\
\hline Total Count & 55 & 55 & 55 & 0 \\
\hline Height $/ \mathrm{nm}$ & 2.0 & 1.1 & 8.0 & 1.1 \\
\hline Area $/ \mathrm{nm}^{2}$ & 2404.1 & 488.3 & 19882.8 & 4068.8 \\
\hline Diameter / nm & 46.8 & 24.9 & 159.1 & 29.6 \\
\hline
\end{tabular}


Table S7. Calculated IAST selectivities (at 1 bar) of COFs for equimolar binary gas mixtures.

\begin{tabular}{|c|c|c|c|}
\hline COFs & Temperature (K) & $\mathrm{CO}_{2} / \mathrm{CH}_{4}$ & $\mathrm{CO}_{2} / \mathrm{H}_{2}$ \\
\hline NUS-2 & 273 & 58.8 & 116.8 \\
\hline NUS-2 & 298 & 29.1 & 53.6 \\
\hline NUS-3 & 273 & 29.3 & 22.0 \\
\hline NUS-3 & 298 & 18.2 & 17.2 \\
\hline
\end{tabular}


Table S8. Pure component $\mathrm{CO}_{2}$ and $\mathrm{CH}_{4}$ permeation properties of Ultem membrane and Ultem-containing MMMs measured at $35{ }^{\circ} \mathrm{C}$ under various pressures.

\begin{tabular}{|c|c|c|c|c|c|c|}
\hline Membrane & $\begin{array}{l}\text { Pressure } \\
\text { (bar) }\end{array}$ & $\begin{array}{c}\mathrm{H}_{2} \\
\text { Permeability } \\
\text { (Barrer) }\end{array}$ & $\begin{array}{c}\mathrm{CO}_{2} \\
\text { Permeability } \\
\text { (Barrer) }\end{array}$ & $\begin{array}{c}\mathrm{CH}_{4} \\
\text { Permeability } \\
\text { (Barrer) }\end{array}$ & $\begin{array}{c}\text { Ideal } \\
\text { Selectivity } \\
\mathrm{H}_{2} / \mathrm{CO}_{2}\end{array}$ & $\begin{array}{c}\text { Ideal } \\
\text { Selectivity } \\
\mathrm{CO}_{2} / \mathrm{CH}_{4}\end{array}$ \\
\hline \multirow{3}{*}{ Ultem } & 2 & $6.39 \pm 0.02$ & $2.22 \pm 0.01$ & $0.11 \pm 0.00$ & 2.88 & 20.2 \\
\hline & 3.5 & $6.38 \pm 0.02$ & $2.08 \pm 0.05$ & $0.11 \pm 0.01$ & 3.07 & 18.9 \\
\hline & 5 & $6.45 \pm 0.02$ & $2.01 \pm 0.01$ & $0.10 \pm 0.01$ & 3.21 & 20.1 \\
\hline \multirow{3}{*}{$\begin{array}{c}10 w t \% \\
\text { NUS-2@Ultem }\end{array}$} & 2 & $12.7 \pm 0.1$ & $3.75 \pm 0.01$ & $0.15 \pm 0.01$ & 3.39 & 25.0 \\
\hline & 3.5 & $12.9 \pm 0.1$ & $3.67 \pm 0.01$ & $0.16 \pm 0.01$ & 3.51 & 22.9 \\
\hline & 5 & $13.4 \pm 0.1$ & $3.76 \pm 0.01$ & $0.16 \pm 0.01$ & 3.56 & 23.5 \\
\hline \multirow{3}{*}{$\begin{array}{c}20 w t \% \\
\text { NUS-2@Ultem }\end{array}$} & 2 & $22.7 \pm 0.1$ & $4.92 \pm 0.02$ & $0.22 \pm 0.01$ & 4.61 & 22.4 \\
\hline & 3.5 & $22.5 \pm 0.3$ & $4.41 \pm 0.04$ & $0.13 \pm 0.01$ & 5.10 & 33.9 \\
\hline & 5 & $22.5 \pm 0.1$ & $3.88 \pm 0.01$ & $0.12 \pm 0.01$ & 5.80 & 32.3 \\
\hline \multirow{3}{*}{$\begin{array}{c}30 w t \% \\
\text { NUS-2@Ultem }\end{array}$} & 2 & $16.5 \pm 0.1$ & $8.70 \pm 0.01$ & $0.69 \pm 0.01$ & 1.89 & 12.7 \\
\hline & 3.5 & $15.0 \pm 0.1$ & $8.54 \pm 0.03$ & $0.51 \pm 0.03$ & 1.76 & 16.9 \\
\hline & 5 & $16.6 \pm 0.1$ & $7.98 \pm 0.01$ & $0.48 \pm 0.01$ & 2.08 & 16.7 \\
\hline \multirow{3}{*}{$\begin{array}{c}10 w t \% \\
\text { NUS-3@Ultem }\end{array}$} & 2 & $14.5 \pm 0.2$ & $5.89 \pm 0.05$ & $0.26 \pm 0.00$ & 2.46 & 22.7 \\
\hline & 3.5 & $13.3 \pm 0.2$ & $5.79 \pm 0.03$ & $0.29 \pm 0.01$ & 2.30 & 20.0 \\
\hline & 5 & $13.6 \pm 0.1$ & $5.68 \pm 0.03$ & $0.28 \pm 0.01$ & 2.39 & 20.3 \\
\hline \multirow{3}{*}{$\begin{array}{c}20 w t \% \\
\text { NUS-3@Ultem }\end{array}$} & 2 & $33.4 \pm 0.2$ & $15.0 \pm 0.2$ & $0.53 \pm 0.01$ & 2.23 & 28.3 \\
\hline & 3.5 & $33.3 \pm 0.1$ & $15.0 \pm 0.1$ & $0.50 \pm 0.01$ & 2.23 & 30.0 \\
\hline & 5 & $33.2 \pm 0.3$ & $13.9 \pm 0.0$ & $0.49 \pm 0.02$ & 2.39 & 28.4 \\
\hline \multirow{3}{*}{$\begin{array}{c}30 w t \% \\
\text { NUS-3@Ultem }\end{array}$} & 2 & $19.9 \pm 0.2$ & $8.11 \pm 0.04$ & $0.76 \pm 0.02$ & 2.45 & 10.7 \\
\hline & 3.5 & $20.4 \pm 0.2$ & $8.17 \pm 0.02$ & $0.80 \pm 0.01$ & 2.50 & 10.2 \\
\hline & 5 & $20.8 \pm 0.1$ & $8.38 \pm 0.01$ & $0.97 \pm 0.02$ & 2.48 & 8.64 \\
\hline \multirow{3}{*}{$\begin{array}{c}20 w t \% \\
\text { NUS-2@Ultem } \\
\text { without sonication }\end{array}$} & 2 & $9.83 \pm 0.02$ & $6.51 \pm 0.02$ & $0.25 \pm 0.01$ & 1.51 & 26.0 \\
\hline & 3.5 & $9.91 \pm 0.01$ & $6.31 \pm 0.05$ & $0.26 \pm 0.01$ & 1.57 & 24.3 \\
\hline & 5 & $10.0 \pm 0.1$ & $6.75 \pm 0.1$ & $0.26 \pm 0.01$ & 1.48 & 26.0 \\
\hline
\end{tabular}


Table S9. Pure component $\mathrm{H}_{2}$ and $\mathrm{CO}_{2}$ permeation properties of PBI membrane and $\mathrm{PBI}$-containing MMMs measured at $35{ }^{\circ} \mathrm{C}$ under various pressures.

\begin{tabular}{|c|c|c|c|c|}
\hline Membrane & $\begin{array}{l}\text { Pressure } \\
\text { (bar) }\end{array}$ & $\begin{array}{c}\mathrm{H}_{2} \text { Permeability } \\
\text { (Barrer) }\end{array}$ & $\begin{array}{c}\mathrm{CO}_{2} \text { Permeability } \\
\text { (Barrer) }\end{array}$ & $\begin{array}{l}\text { Ideal Selectivity } \\
\qquad \mathrm{H}_{2} / \mathrm{CO}_{2}\end{array}$ \\
\hline \multirow{3}{*}{ PBI } & 2 & $3.62 \pm 0.02$ & $0.40 \pm 0.01$ & 9.05 \\
\hline & 3.5 & $3.62 \pm 0.02$ & $0.39 \pm 0.02$ & 9.28 \\
\hline & 5 & $3.61 \pm 0.01$ & $0.38 \pm 0.02$ & 9.50 \\
\hline \multirow{3}{*}{ 10wt\%NUS-2@PBI } & 2 & $6.25 \pm 0.01$ & $0.59 \pm 0.01$ & 10.6 \\
\hline & 3.5 & $6.25 \pm 0.01$ & $0.57 \pm 0.01$ & 11.0 \\
\hline & 5 & $6.24 \pm 0.01$ & $0.50 \pm 0.01$ & 12.5 \\
\hline \multirow{3}{*}{ 20wt\%NUS-2@PBI } & 2 & $4.07 \pm 0.02$ & $0.32 \pm 0.02$ & 12.7 \\
\hline & 3.5 & $4.00 \pm 0.01$ & $0.21 \pm 0.02$ & 19.0 \\
\hline & 5 & $4.08 \pm 0.03$ & $0.13 \pm 0.01$ & 31.4 \\
\hline \multirow{3}{*}{ 30wt\%NUS-2@PBI } & 2 & $3.91 \pm 0.01$ & $0.34 \pm 0.01$ & 11.4 \\
\hline & 3.5 & $3.91 \pm 0.02$ & $0.34 \pm 0.01$ & 11.5 \\
\hline & 5 & $3.78 \pm 0.01$ & $0.30 \pm 0.01$ & 12.6 \\
\hline \multirow{3}{*}{ 10wt\%NUS-3@PBI } & 2 & $7.74 \pm 0.02$ & $0.99 \pm 0.01$ & 7.82 \\
\hline & 3.5 & $7.49 \pm 0.01$ & $0.89 \pm 0.01$ & 8.42 \\
\hline & 5 & $7.32 \pm 0.01$ & $0.86 \pm 0.01$ & 8.51 \\
\hline \multirow{3}{*}{ 20wt\%NUS-3@PBI } & 2 & $12.3 \pm 0.1$ & $1.55 \pm 0.01$ & 7.94 \\
\hline & 3.5 & $12.1 \pm 0.1$ & $1.49 \pm 0.03$ & 8.12 \\
\hline & 5 & $12.2 \pm 0.1$ & $1.37 \pm 0.02$ & 8.91 \\
\hline \multirow{3}{*}{ 30wt\%NUS-3@PBI } & 2 & $52.4 \pm 0.1$ & $13.9 \pm 0.1$ & 3.77 \\
\hline & 3.5 & $60.9 \pm 0.1$ & $17.6 \pm 0.1$ & 3.47 \\
\hline & 5 & $63.1 \pm 0.1$ & $18.9 \pm 0.1$ & 3.33 \\
\hline
\end{tabular}


Table S10. Equimolar $\mathrm{H}_{2} / \mathrm{CO}_{2}$ mixed gas permeation tests of MMMs containing 20wt $\%$ COFs measured at 5 bar and $35^{\circ} \mathrm{C}$.

\begin{tabular}{|l|l|l|l|l|}
\hline \multicolumn{1}{|c|}{ Membrane } & $\begin{array}{c}\mathrm{H}_{2} \text { Permeability } \\
\text { (Barrer) }\end{array}$ & $\begin{array}{c}\mathrm{CO}_{2} \text { Permeability } \\
\text { (Barrer) }\end{array}$ & $\begin{array}{c}\text { Ideal Selectivity } \\
\mathrm{H}_{2} / \mathrm{CO}_{2}\end{array}$ & $\begin{array}{c}\text { Separation } \\
\text { Factor } \\
\mathrm{H}_{2} / \mathrm{CO}_{2}\end{array}$ \\
\hline NUS-2@Ultem & $17.11 \pm 0.06$ & $3.40 \pm 0.01$ & 5.80 & 5.04 \\
\hline NUS-2@PBI & $2.97 \pm 0.03$ & $0.16 \pm 0.001$ & 31.40 & 18.78 \\
\hline NUS-3@Ultem & $23.89 \pm 0.04$ & $6.87 \pm 0.01$ & 2.39 & 3.48 \\
\hline NUS-3@PBI & $10.04 \pm 0.07$ & $1.13 \pm 0.006$ & 8.91 & 8.91 \\
\hline
\end{tabular}

